\title{
PGC-1 $\alpha$ ameliorates kidney fibrosis in mice with diabetic kidney disease through an antioxidative mechanism
}

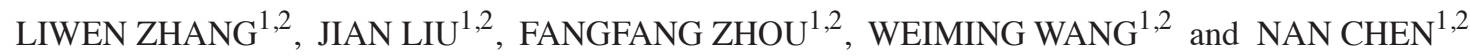 \\ ${ }^{1}$ Department of Nephrology, Ruijin Hospital; ${ }^{2}$ Institute of Nephrology, \\ Shanghai Jiao Tong University School of Medicine, Shanghai 200025, P.R. China
}

Received May 8, 2017; Accepted December 7, 2017

DOI: $10.3892 / \mathrm{mmr} .2018 .8433$

\begin{abstract}
The production of reactive oxygen species (ROS) is a common phenomenon in podocyte impairment, which leads to the irreversible progression of chronic kidney diseases, such as diabetic kidney disease (DKD). Previous research has indicated that peroxisome proliferator-activated receptor $\gamma(\mathrm{PPAR} \gamma)$ coactivator-1 $\alpha$ (PGC-1 $\alpha)$ participates in mitochondrial biogenesis and energy metabolism in certain mitochondria-enriched cells, including myocardial and skeletal muscle cells. Therefore, we hypothesized that PGC-1 $\alpha$ may be a protective nuclear factor against energy and oxidative stress in DKD. To investigate this hypothesis, $\mathrm{db} / \mathrm{db}$ diabetic mice were used to establish a DKD model and the PPAR $\gamma$ agonist rosiglitazone was employed to induce PGC- $1 \alpha$ expression in vivo. Additionally, immortalized mouse podocytes and SV40 MES 13 renal mesangial cells were utilized for in vitro experiments. The expression levels of PGC- $1 \alpha$ and genes associated with kidney and cell injury were determined by western blotting or reverse transcription-quantitative polymerase chain reaction and intracellular ROS levels were assessed by 2',7'-dichlorodihydrofluorescein diacetate. The results of the present study demonstrated that endogenous PGC-1 $\alpha$ expression exhibited protective effects against oxidative stress, glomerulosclerosis and tubulointerstitial fibrosis in experimental DKD. These results indicated a potential role of PGC-1 $\alpha$ in the amelioration of key pathophysiological features of DKD and provided evidence for PGC- $1 \alpha$ as a potential therapeutic target in DKD.
\end{abstract}

\section{Introduction}

Diabetic kidney disease (DKD) is the primary cause of end-stage kidney disease (ESRD) in western countries, and

Correspondence to: Professor Weiming Wang, Department of Nephrology, Ruijin Hospital, Shanghai Jiao Tong University School of Medicine, 197 Ruijin Er Road, Shanghai 200025, P.R. China E-mail: wweiming@medmail.com.cn

Key words: peroxisome proliferator-activated receptor $\gamma$ coactivator-1 $\alpha$, diabetic kidney disease, oxidative damage $\sim 43.8 \%$ of patients with ESRD have diabetes, according to a US renal data system report (1). The incidence rate of DKD has surpassed that of glomerulonephritis, which has led to DKD becoming the major causative factor of ESRD in China (2) and an increasingly predominant cause of ESRD in other developing countries.

Long-term hyperglycemia in patients with diabetes leads to intracellular glucose overload and energy stress. As a result, a number of signaling pathways, including transforming growth factor- $\beta$ (TGF- $\beta$ )-mothers against decapentaplegic homolog 2-mitogen-activated protein kinase 1 and tyrosine-protein kinase JAK/signal transducer and activator of transcription, are activated via similar mechanisms in different types of renal cells (3). The activation of the aforementioned pathways leads to the impairment of mitochondrial structure and function in kidney cells, which in turn leads to increased reactive oxygen species (ROS) synthesis. The subsequent oxidative stress is a key factor in podocyte and mesangial cell impairment and is a common mechanism in multiple pathways that lead to DKD occurrence and progression (3).

Early stage DKD is characterized by an increased glomerular filtration rate, transient microalbuminuria, podocyte and mesangial cell impairment, glomerular basement membrane (GBM) thickening and extracellular matrix accumulation in the mesangium. Increasing evidence indicates important roles for TGF- $\beta$ in energy and metabolic balance, mitochondrial stability and ROS synthesis $(4,5)$, which are major contributors to podocyte depletion and kidney fibrosis.

A previous report demonstrated that peroxisome proliferator-activated receptor $\gamma(\operatorname{PPAR} \gamma)$ coactivator-1 $\alpha$ (PGC-1 $\alpha)$ participates in mitochondrial biogenesis in tissues with high energy consumption, including skeletal muscle and the heart, as a key nuclear factor in energy and oxidative stress (6). PGC-1 $\alpha$ is also highly expressed in the kidney $(7,8)$ and is reported to protect against several kidney diseases. Specifically, PGC-1 $\alpha$ upregulation has been demonstrated to alleviate mitochondrial dysfunction in acute kidney injury and an adriamycin nephrosis model $(9,10)$. However, to the best of our knowledge, the role of PGC-1 $\alpha$ in DKD is yet to be established.

PPAR $\gamma$ agonists, as major pharmaceutical inducers of PGC-1 $\alpha$, have been widely employed to investigate the mechanisms of PGC-1 $\alpha$ (11-13). In the present study, the PPAR $\gamma$ agonist rosiglitazone was used in a $\mathrm{db} / \mathrm{db}$ mouse model of DKD to investigate the effects of PGC-1 $\alpha$ in vivo. 
Furthermore, genetic regulation of PGC-1 $\alpha$ was investigated in renal mesangial cells to investigate the underlying mechanisms in vitro. The results of the current study revealed a novel association between PGC-1 $\alpha$ expression and DKD progression and the underlying mechanisms. These observations indicate that endogenous PGC-1 $\alpha$ may have potential as an effective therapeutic target in DKD.

\section{Materials and methods}

Animal experiments. Animal maintenance and experimental procedures were approved by the Animal Care Committee of Ruijin Hospital, Shanghai Jiao Tong University School of Medicine (Shanghai, China). A total of 12 male $\mathrm{db} / \mathrm{db}$ diabetic mice weighing 32-34 g with a C57BL/KsJ (BKS. Cg-Dock $7^{\mathrm{m}+/+}$ Lepr $^{\mathrm{db}} / \mathrm{Nju}$ ) background and 6 male non-diabetic littermate control $\mathrm{db} / \mathrm{m}$ mice weighing $16-18 \mathrm{~g}$ (6 weeks old) were obtained from Nanjing Biomedical Research Institute of Nanjing University (Nanjing, China). Mice were separated into three groups ( $\mathrm{n}=6 /$ group) as follows: $\mathrm{db} / \mathrm{m}$ (control); db/db (DKD group) and $\mathrm{db} / \mathrm{db}$ mice administered $20 \mathrm{mg} / \mathrm{kg} /$ day rosiglitazone (Sigma-Aldrich; Merck KGaA, Darmstadt, Germany; RGZ group) by gavage for 8 weeks. Mice were housed in a specific pathogen-free room at a constant temperature of $22 \pm 2^{\circ} \mathrm{C}$ and a constant humidity of $50 \pm 5 \%$, normal air $\mathrm{CO}_{2}$ and a 12-h light/dark cycle and kept with free access to chow and water. Mice were sacrificed after the treatment for 8 weeks.

Immortalized mouse podocyte culture. Conditionally immortalized mouse podocytes were kindly provided by Professor John Cijiang He (Department of Nephrology, Icahn School of Medicine at Mount Sinai, New York City, NY, USA) and cultured as previously described (14). Briefly, cells were cultured on Corning Collagen I plates (Corning Incorporated, Corning, NY, USA) at $33^{\circ} \mathrm{C}$ in RPMI 1640 medium (Gibco; Thermo Fisher Scientific, Inc., Waltham, MA, USA) with the presence of $20 \mathrm{U} / \mathrm{ml}$ mouse recombinant interferon (IFN)- $\gamma$ (R\&D Systems, Inc., Minneapolis, MN, USA) and 10\% fetal bovine serum (FBS; Gibco; Thermo Fisher Scientific, Inc.) to enhance the expression of thermosensitive $\mathrm{T}$ antigen. Immortalized mouse podocyte cell lines are routinely characterized in the laboratory on the basis of morphology and gene expression patterns, prior to the experimentation. Immortalized cells for all experiments in the current study were between passages 4 and 12. To induce differentiation, podocytes were maintained in RPMI medium and FBS at $37^{\circ} \mathrm{C}$ without IFN- $\gamma$ for $10-12$ days. Podocytes prepared for experiments were serum deprived for $24 \mathrm{~h}$ prior to intervention, followed by culture with D-glucose (Sigma-Aldrich; Merck KGaA) or D-mannitol (Sigma-Aldrich; Merck KGaA) added to the cultures at $30 \mathrm{mM}$ glucose as high-glucose stimulation or at $5.5 \mathrm{mM}$ glucose and $24.5 \mathrm{mM}$ mannitol as isotonic control for 24 or $48 \mathrm{~h}$.

Mouse mesangial cell culture and transfection. SV40 MES 13 mouse mesangial cells were obtained from the American Type Culture Collection (Manassas, VA, USA) and maintained in 3:1 mixture of Dulbecco's modified Eagle's medium (Gibco; Thermo Fisher Scientific, Inc.) and Ham's F12 medium with
14 mM HEPES (Gibco; Thermo Fisher Scientific, Inc.) and 5\% FBS (Gibco; Thermo Fisher Scientific, Inc.) as full medium at $37^{\circ} \mathrm{C}$ with $5 \% \mathrm{CO}_{2}$. The overexpression plasmid for PGC-1 $\alpha$ and its negative control were gifts from Professor Zhiguo Zhang (State Key Laboratory of Medical Genomics, Shanghai Institute of Endocrine and Metabolic Diseases, Shanghai Clinical Center for Endocrine and Metabolic Diseases, Ruijin Hospital, Shanghai Jiaotong University School of Medicine, Shanghai, China) (15). Mesangial cells were seeded at $3 \times 10^{5}$ cells/well and were transfected using Lipofectamine ${ }^{\mathrm{TM}}$ 3000 (Invitrogen; Thermo Fisher Scientific, Inc.) with $2.5 \mu \mathrm{g}$ plasmid DNA/well, according to the manufacturer's protocol. A total of $24 \mathrm{~h}$ following transfection, cells were stimulated at the concentration of $30 \mathrm{mM}$ D-glucose (high glucose) or $5.5 \mathrm{mM}$ D-glucose + $24.5 \mathrm{mM}$ D-mannitol (isotonic control) for $48 \mathrm{~h}$.

Lentivirus production and cell infection. The lentivirus suppressing PGC-1 $\alpha$ was produced with the short hairpin (sh)RNA sequence 5'-TCCAGTAAGCACACGTTTATT-3'. A scrambled shRNA sequence 5'-TTCTCCGAACGTGTC ACGT-3' was used as the negative control for shRNA-PGC-1 $\alpha$. Double-strand oligonucleotides were inserted into the linear pLKO.1 vector (Genechem, Shanghai, China) to produce the lentivirus. Briefly, two pairs of DNA primer fragments with AgeI and EcoR I restriction sites were synthesized and annealed to form double strands, which were ligated with linear pLKO.1 vector by $\mathrm{T} 4$ ligase and confirmed by sequencing, respectively. The Lenti-Easy Packaging System (cat. no. LPK001, Genechem) was applied to generate the packaged virus with 293T cell line (American Type Culture Collection) according to the manufacturers' protocol. The SV40 MES 13 mouse mesangial cells were seeded at $3 \times 10^{5}$ cells/well in a 6 -well plate with full medium. The next day cells were washed with PBS and left for $2 \mathrm{~h}$ in $1 \mathrm{ml}$ serum-free medium, each well containing $2 \times 10^{6}$ viral particles (the appropriate number of viral particles were determined by a preliminary experiment, using puromycin screening). Subsequently $1 \mathrm{ml}$ full medium was added in each well and the medium was replaced with fresh full medium after $24 \mathrm{~h}$. A total of $72 \mathrm{~h}$ following-infection, the expression of PGC-1 $\alpha$ in infected SV40 MES 13 cells was confirmed by reverse transcription-quantitative polymerase chain reaction (RT-qPCR) and D-glucose or D-mannitol treatment (at the concentration of $30 \mathrm{mM} \mathrm{D}$-glucose or $5.5 \mathrm{mM}$ D-glucose $+24.5 \mathrm{mM}$ D-mannitol for $48 \mathrm{~h}$ ) was administered.

Biochemical analysis of serum and urine samples. Albumin concentration in urine was detected using Mouse Albumin ELISA Quantitation Set (cat. no. E90-134) (Bethyl Laboratories, Inc., Montgomery, TX, USA) and glucose concentration in serum was detected by Glucose LiquiColor ${ }^{\circledR}$ (Enzymatic) test kit (Stanbio Laboratory; EFK Diagnostics, Inc., Boerne, TX, USA), according to the manufacturers' protocols. After 8 weeks of treatment, urine was collected over $24 \mathrm{~h}$ in metabolic cages to measure and calculate the urinary albumin excretion (UAE). Blood from the caudal vein was collected for monitoring serum glucose weekly. Briefly, $200 \mu \mathrm{l}$ blood was collected into an Eppendorf tube and placed at $4^{\circ} \mathrm{C}$ for $1 \mathrm{~h}$ until blood coagulation. Subsequently the blood was 
centrifuged at $3,000 \mathrm{xg}, 4^{\circ} \mathrm{C}$ for $10 \mathrm{~min}$ and serum supernatant was collected and stored at $-80^{\circ} \mathrm{C}$ prior to analysis.

Mitochondrial superoxide dismutase activity assay. Mitochondrial fractions of kidney cortexes of the mice were isolated using Minute ${ }^{\mathrm{TM}}$ Mitochondria Isolation kit for mammalian cells and tissues (cat. no. MP-007; Invent Biotechnologies, Inc., Plymouth, MN, USA), according to the manufacturer's protocol. The isolated mitochondria were solubilized in Non-denatured Protein Solubilization reagent (Invent Biotechnologies, Inc.) for downstream superoxide dismutase (SOD) activity determination. SOD activity was detected using SOD assay kit (WST-1 method; cat. no. A001-3; Jiancheng Bioengineering Institute, Nanjing, China) and an iMark $^{\mathrm{TM}}$ Microplate Reader (Bio-Rad Laboratories, Inc., Hercules, CA, USA), according to the manufacturers' protocols.

Kidney histopathology. Kidneys removed from euthanized mice and were immediately cut in half, fixed in $10 \%$ formaldehyde in $0.1 \mathrm{~mol} / 1 \mathrm{PBS}\left(\mathrm{pH} \mathrm{7.2)}\right.$ at $4^{\circ} \mathrm{C}$ for $24 \mathrm{~h}$, then embedded in paraffin and sectioned at $4 \mu \mathrm{m}$. The $4-\mu \mathrm{m}$-sections were dewaxed in xylene, rehydrated through decreasing concentrations of ethanol and washed in PBS. Subsequently, for each sample, one section was stained with Harris hematoxylin staining solution for $5 \mathrm{~min}$ and eosin staining solution for $2 \mathrm{~min}$ (Goodbio Technology CO., LTD, Wuhan, China) as H\&E staining. For each sample, another section was stained with Weigert's iron hematoxylin for 5 min, Ponceau S for 6 min, molybdphosphoric acid resolution for $3 \mathrm{~min}$ and aniline blue for $5 \mathrm{~min}$, as Masson's trichrome staining. All aforementioned staining steps were performed at room temperature $\left(23^{\circ} \mathrm{C}\right)$. Following staining, sections were dehydrated through increasing concentrations of ethanol and xylene. The general histological alterations in glomerular and tubular structures were evaluated under a light microscope.

Western blot analysis. Nuclear and cytosolic fractions of the kidney cortexes were separated using NE-PER ${ }^{\mathrm{TM}}$ Nuclear and Cytoplasmic Extraction reagents (Applied Biosystems; Thermo Fisher Scientific, Inc.) and cells were lysed in radioimmunoprecipitation assay buffer (Beyotime Institute of Biotechnology, Haimen, China) containing protease inhibitor cocktail (Bimake, Houston, TX, USA) to extract the total protein. The concentration of proteins was determined by Bicinchoninic Acid (BCA) method using BCA Protein Assay kit (cat. no. ZJ101; Shanghai Epizyme Biotechnology Co., Ltd., Shanghai, China). A total of $30 \mu \mathrm{g}$ cytoplasmic or total protein sample or $20 \mu \mathrm{g}$ nuclear protein sample was loaded per lane. Electrophoretic analysis of proteins was carried out on 10\% SDS-PAGE gel and transferred onto polyvinylidene difluoride membranes (EMD Millipore, Billerica, MA, USA). Membranes were blocked with QuickBlock ${ }^{\mathrm{TM}}$ Blocking Buffer (cat. no. P0252; Beyotime Institute of Biotechnology) at room temperature on a horizontal shaker for $10 \mathrm{~min}$. Subsequently the membranes were incubated with the primary antibodies overnight at $4^{\circ} \mathrm{C}$. For nuclear or cytoplasmic proteins of renal cortex tissues and total proteins of SV40 MES 13 mouse mesangial cells anti-PGC-1 $\alpha$ (cat. no. ab3242; 1:1,000; EMD Millipore) was used. For cytoplasmic proteins of renal cortex tissues, anti-E-cadherin (cat. no. ab76055; 1:1,000; Abcam, Cambridge, MA, USA), anti-collagen type $1 \alpha 1$ (COL1 $\alpha 1$; cat. no. sc-8784; 1:200; Santa Cruz Biotechnology, Inc., Dallas, TX, USA) and anti- $\alpha$-smooth muscle actin ( $\alpha$-SMA; cat. no. sc-53015; 1:300; Santa Cruz Biotechnology, Inc.) were used. For total proteins of mice podocytes, anti-nephrin (cat. no. ab58968; 1:300; Abcam), anti-synaptopodin (cat. no. sc-21537; 1:300; Santa Cruz Biotechnology, Inc.) and anti-cleaved-caspase-3 (cat. no. 9661; 1:1,000; Cell Signaling Technology, Inc., Danvers, MA, USA) were used. Anti-histone H2A.X (cat. no. 7631; 1:1,000; Cell Signaling Technology, Inc.) and anti- $\beta$-actin (cat. no. ab8226; 1:2,000; Abcam) were used as the loading controls for nuclear and cytoplasmic/total protein expression, respectively. Subsequently, membrane were incubated with anti-mouse immunoglobulin $(\mathrm{Ig}) \mathrm{G}$, horseradish peroxidase (HRP)-conjugated antibody (cat. no. 7076; 1:2,000; Cell Signaling Technology, Inc.) or anti-rabbit IgG, HRP-conjugated antibody (cat. no. 7074; 1:2,000; Cell Signaling Technology, Inc.) for $1 \mathrm{~h}$ at room temperature on a horizontal shaker. The bands were detected using Immobilon Western Chemiluminescent HRP Substrate (cat. no. WBKLS0100; EMD Millipore) and band intensities were quantified using ImageJ (version number 1.8.0; National Institutes of Health, Bethesda, MD, USA).

$R T-q P C R$. Total RNA from mouse podocytes and SV40 MES 13 mesangial cells was extracted using TRIzol reagent (Thermo Fisher Scientific, Inc.). RNA concentration was determined with an ND-1000 spectrophotometer (NanoDrop Technologies; Thermo Fisher Scientific, Inc.). First-strand cDNA synthesis was performed using $2 \mu \mathrm{g}$ RNA with High-Capacity cDNA Reverse Transcription kit with RNase Inhibitor (Applied Biosystems; Thermo Fisher Scientific, Inc.), according to the manufacturer's protocol. To test for genomic DNA contamination, additional reactions were performed without the addition of reverse transcriptase. qPCR was performed using the SYBR ${ }^{\circledR}$ Green PCR Master Mix (Applied Biosystems; Thermo Fisher Scientific, Inc.) and the StepOnePlus Real-Time PCR System (Applied Biosystems; Thermo Fisher Scientific, Inc.) using the two-step method and the following reaction conditions: Initial denaturation at $95^{\circ} \mathrm{C}$ for $10 \mathrm{~min} ; 40$ cycles of $95^{\circ} \mathrm{C}$ for $15 \mathrm{sec}, 60^{\circ} \mathrm{C}$ for $60 \mathrm{sec}$, according to manufacturer's protocol. The primer sequences used are presented in Table I. mRNA expression was normalized to $\beta$-actin and quantified using the comparative quantification cycle $\left(2^{-\Delta \Delta \mathrm{Cq}}\right)$ method (16).

Measurement of intracellular ROS. Intracellular ROS levels were measured using an ROS assay kit (Beyotime Institute of Biotechnology). Following overexpression or knockdown of PGC-1 $\alpha$, SV40 MES 13 cells were treated with $30 \mathrm{mM}$ D-glucose or 5.5 mM D-glucose + $24.5 \mathrm{mM} \mathrm{D}$-mannitol for $48 \mathrm{~h}$ at $37^{\circ} \mathrm{C}$ and subsequently replaced by fresh FBS-free medium for mesangial cells (mentioned above) with $10 \mu \mathrm{M}$ 2',7'-dichlorodihydrofluorescein diacetate (DCFH-DA) in Reactive Oxygen Species Assay kit (Beyotime Institute of Biotechnology) to stain for $60 \mathrm{~min}$ at $37^{\circ} \mathrm{C}$. Following washing twice, fluorescence was measured $(485 \mathrm{~nm}$ excitation $/ 528 \mathrm{~nm}$ emission) with a Synergy 2 fluorescence microplate reader (BioTek Instruments, Inc., Winooski, VT, USA). 
Table I. Primer sequences used in the reverse transcription-quantitative polymerase chain reaction to detect mRNA expression.

\begin{tabular}{lll}
\hline Gene & \multicolumn{1}{c}{ Forward primer $\left(5^{\prime}-3^{\prime}\right)$} & \multicolumn{1}{c}{ Reverse primer $\left(5^{\prime}-3^{\prime}\right)$} \\
\hline NDUFS1 & CACCCATTGGATTGTCCTATTT & ACAGCACGCTTCCCCTCT \\
COX4I1 & GCTTTCCCCACTTACGCTG & TTGGTGCCCCTGTTCATCT \\
PGC-1 $\alpha$ & TGACAGATGGAGCCGTGACC & TGTGGGTGTGGTTTGCTG \\
PPAR $\gamma$ & TTTCAAGGGTGCCAGTTTCG & GGGAGGCCAGCATCGTGT \\
PPRC1 & ACTGCTTGCCCAACCTCAC & ATCTGCCGCACCACGAC \\
NRF1 & TCGTACCATCACAGACCGTAGT & GCCCAGTTTGTTCCACCT \\
\hline
\end{tabular}

NDUFS1, NADH dehydrogenase (ubiquinone) Fe-S protein 1; COX4I1, cytochrome C oxidase subunit 4I1; PPAR $\gamma$, peroxisome proliferatoractivated receptor $\gamma$; PGC-1 $\alpha$, PPAR $\gamma$ coactivator-1 $\alpha$; PPRC1, PGC-1-related coactivator; NRF1, nuclear respiratory factor 1.

$\mathbf{A}$

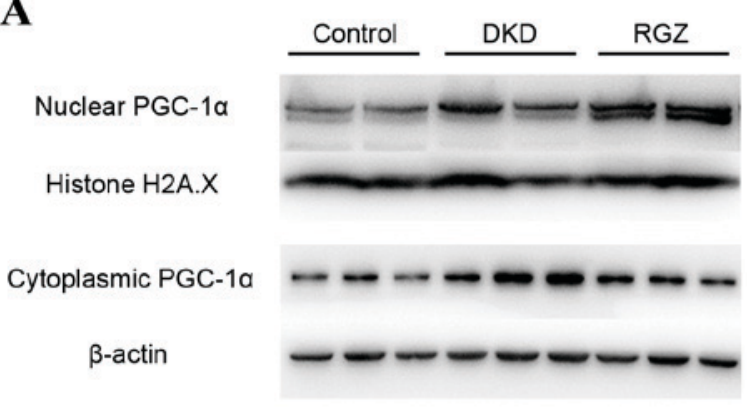

B

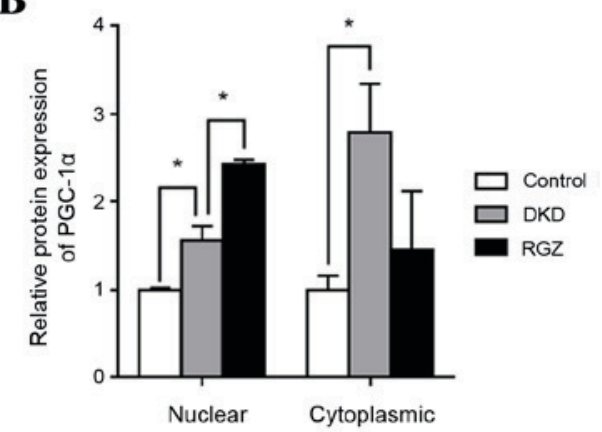

Figure 1. Relative protein expression of PGC-1 $\alpha$ in nuclear and cytoplasmic extractions of the renal cortex of mice. (A) Western blot analysis of PGC-1 $\alpha$ protein expression in the nuclear and cytoplasmic extractions of renal cortexes from mice after 8 weeks treatment. (B) Relative quantification of protein expression from the results of the western blot analysis. Each bar represents the relative mean \pm standard deviation, with the control group expression values set as 1 . $\mathrm{n}=6$ per group. ${ }^{*} \mathrm{P}<0.05$, as indicated. PGC-1 $\alpha$, peroxisome proliferator-activated receptor $\gamma$ coactivator-1 $\alpha$; DKD, diabetic kidney disease; RGZ, rosiglitazone.

Statistical analysis. All experiments were repeated at least three times and data are presented as the mean \pm standard deviation. Statistical analysis was performed using SPSS 22.0 (IBM Corp., Armonk, NY, USA) and significance was calculated with one way analysis of variance followed by Tukey's post-hoc analysis for multiple comparisons, or the Student's t-test for comparisons between two groups. All tests were two tailed. $\mathrm{P}<0.05$ was considered to indicate a statistically significant difference.

\section{Results}

PPAR $\gamma$ agonist induces PGC-1 $\alpha$ expression in the kidney cortex of $\mathrm{db} / \mathrm{db}$ mice. Control mice $(\mathrm{db} / \mathrm{m})$ were considered to exhibit basal expression levels of PGC-1 $\alpha$ protein (Fig. 1). PGC-1 $\alpha$ protein expression was significantly increased in the nuclear and cytoplasmic extractions from db/db kidney cortex compared with the $\mathrm{db} / \mathrm{m}$ controls $(\mathrm{P}<0.05$; Fig. 1$)$. Furthermore, PGC-1 $\alpha$ expression was increased in rosiglitazone-treated DKD mice (RGZ group) compared with the DKD group in the nuclear fraction, but not in the cytoplasmic fraction (Fig. 1).

Pharmacological upregulation of PGC-1 $\alpha$ alleviates DKD in $\mathrm{db} / \mathrm{db}$ mice. Serum glucose levels of the 6 week old $\mathrm{db} / \mathrm{db}$ mice (DKD and RGZ groups) were significantly higher compared with the $\mathrm{db} / \mathrm{m}$ control group (Fig. 2A). However, levels decreased to control levels after 1 week of rosiglitazone administration, while the DKD group exhibited a consistently high serum glucose level throughout the experimental period (Fig. 2A). After the 8 week experimental period (14 week old mice), the UAE of the DKD group was significantly increased compared with the control group, while 8 weeks of rosiglitazone treatment reduced the UAE of DKD mice significantly $(\mathrm{P}<0.05$; Fig. 2B). Additionally, mitochondrial SOD activity was reduced in kidney cortexes of the DKD group compared with the control group $(\mathrm{P}<0.05$; Fig. $2 \mathrm{C})$, while rosiglitazone significantly reversed the inhibition of SOD activity in DKD mice $(\mathrm{P}<0.05$; Fig. 2C).

H\&E staining revealed obvious glomerular hypertrophy and mesangial cell proliferation in the DKD group compared with the control group (Fig. 2D), while Masson's trichome staining revealed substantial mesangial matrix expansion, glomerulosclerosis and tubulointerstitial in the DKD group (Fig. 2E). Rosiglitazone treatment resulted in only minor differences compared with the control group and no marked pathological characteristics were observed (Fig. 2D and E).

Furthermore, western blot analysis revealed a significant decrease in E-cadherin protein expression and an increase in COL1 $\alpha 1$ expression in the renal tissues of the DKD group compared with the control group $(\mathrm{P}<0.01$; Fig. $2 \mathrm{~F}$ and $\mathrm{G})$. 
A

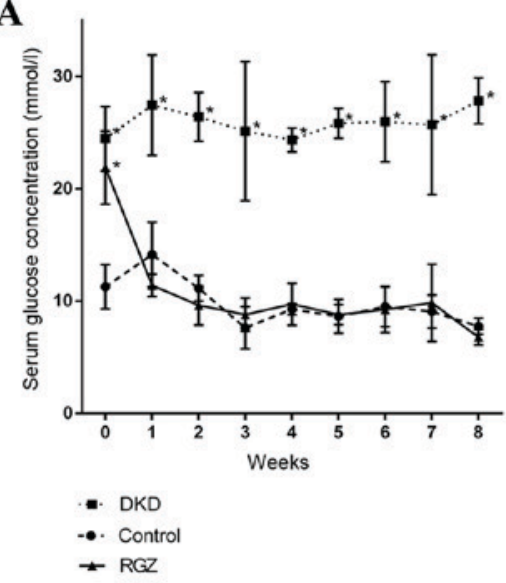

B

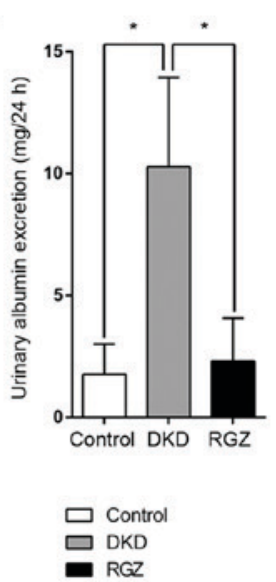

C

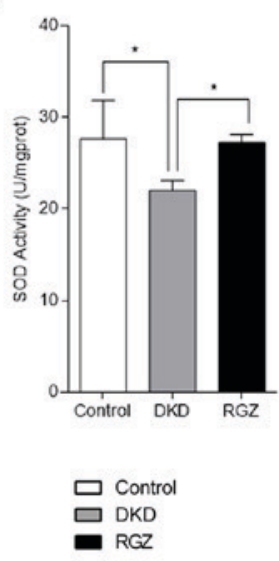

Control

DKD

RGZ
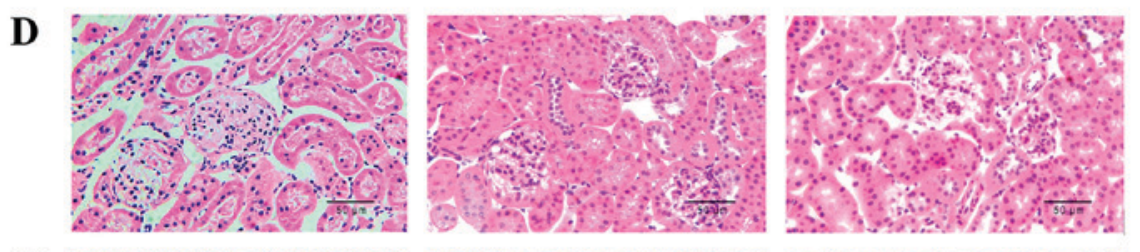

E
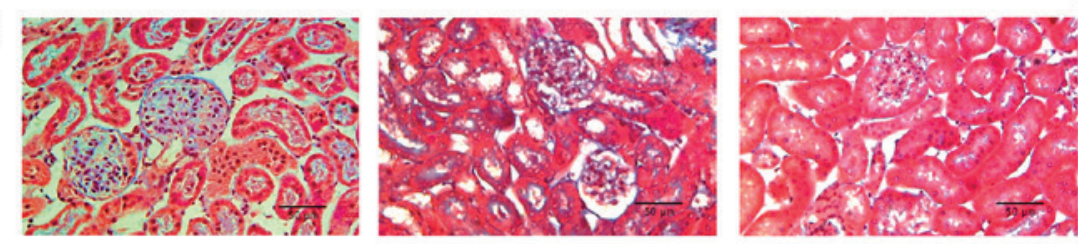

$\mathbf{F}$

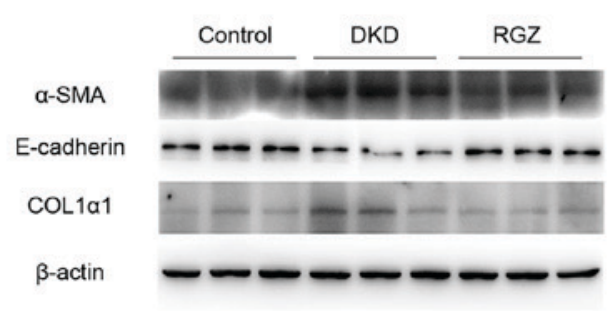

G

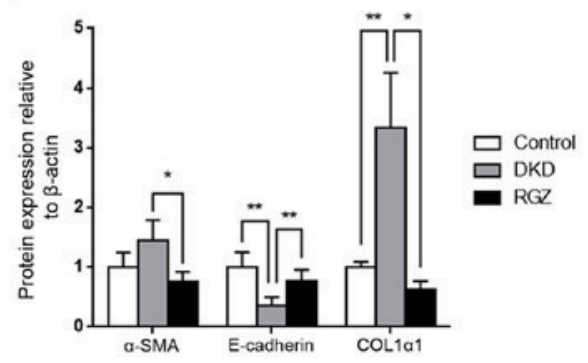

Figure 2. Pharmacological upregulation of PGC-1 $\alpha$ by $20 \mathrm{mg} / \mathrm{kg} /$ day rosiglitazone treatment exhibited protective effects against DKD in mice. (A) Serum glucose levels were detected weekly in the control, DKD and RGZ groups. Data are presented as the mean \pm standard deviation ( $\mathrm{n}=6$ per group). $\mathrm{P}<0.05$ vs. control at the same time point. (B) After 8 weeks of treatment, $24 \mathrm{~h}$ urinary albumin excretion in mice in control, DKD and RGZ groups. $\mathrm{n}=6$ per group. ${ }^{*} \mathrm{P}<0.05$, as indicated. (C) Mitochondrial SOD activity for mice after treatment for 8 weeks ( $\mathrm{n}=6$ per group). ${ }^{*} \mathrm{P}<0.05$, as indicated. Representative photomicrographs depicting (D) hematoxylin and eosin and (E) Masson's trichome staining in the control, DKD and RGZ groups after the 8 week experimental period. Original magnification, $x 400$. (F) Western blot analysis of $\alpha$-SMA, E-cadherin, COL1 $\alpha 1$ and $\beta$-actin protein expression in the renal cortexes of control, DKD and RGZ mice. (G) Densitometric analysis of western blot results. Relative band intensity was normalized to the intensity of the respective $\beta$-actin signal. Data are presented as the mean \pm standard deviation ( $\mathrm{n}=6$ per group). $\mathrm{P}<0.05$ and ${ }^{* *} \mathrm{P}<0.01$, as indicated. $\mathrm{PGC}$ - $1 \alpha$, peroxisome proliferator-activated receptor $\gamma$ coactivator-1 $\alpha$; DKD, diabetic kidney disease; RGZ, rosiglitazone; $\alpha$-SMA, $\alpha$-smooth muscle actin; COL1 $\alpha 1$, collagen type $1 \alpha 1$.

These proteins indicate the occurrence of epithelial-mesenchymal transition (EMT) and fibrosis. However, PGC-1 $\alpha$ upregulation mediated by rosiglitazone counteracted the alterations in $\alpha$-SMA $(\mathrm{P}<0.05)$, E-cadherin $(\mathrm{P}<0.01)$ and COL1A1 $(\mathrm{P}<0.05)$ expression in $\mathrm{db} / \mathrm{db}$ mice $($ Fig. $2 \mathrm{~F}$ and G). Taken together, these data indicate that PGC-1 $\alpha$ upregulation by rosiglitazone in $\mathrm{db} / \mathrm{db}$ mice may lead to the amelioration of key DKD features, including albuminuria, inhibited renal cortex mitochondrial SOD activity and kidney fibrosis.
Impairment of high glucose on mouse podocytes and renal mesangial cells. Following stimulation with high glucose concentrations ( $30 \mathrm{mM}$ ) for $24 \mathrm{~h}, \mathrm{PGC}-1 \alpha$ expression in mouse podocytes increased moderately but significantly $(\mathrm{P}<0.05)$, and PGC-1-related coactivator (PPRC1) was also upregulated $(\mathrm{P}<0.01)$, compared with the $24 \mathrm{~h} \mathrm{D}$-mannitol group (Fig. 3A). However, after $48 \mathrm{~h}$ stimulation with $30 \mathrm{mM}$ glucose, the mRNA expression of PGC-1 $\alpha$, PPRC1, PPAR $\gamma$, downstream nuclear respiratory factor 1 (NRF1), NADH dehydrogenase (ubiquinone) Fe-S protein 1 (NDUFS1) and cytochrome c 
A

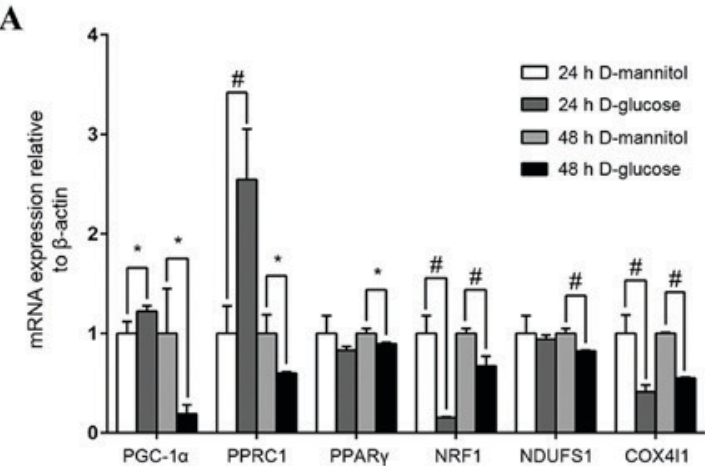

B

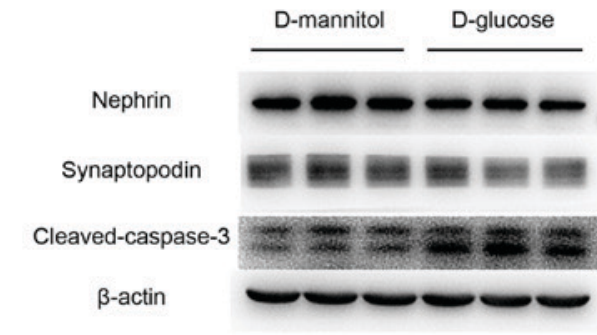

D
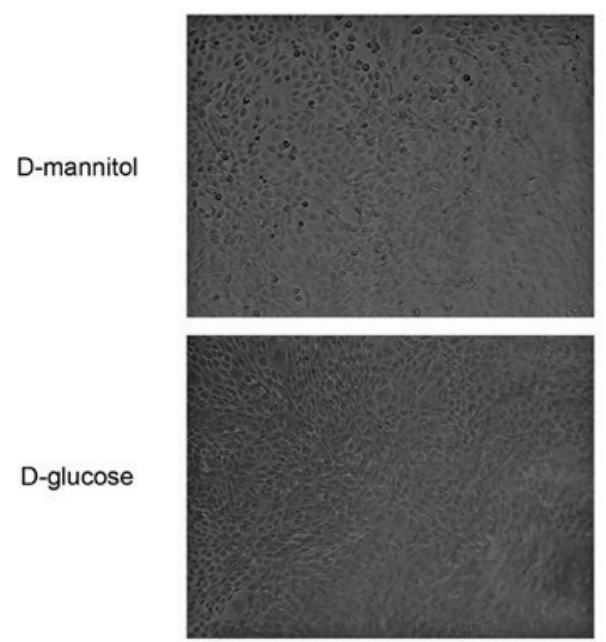

C
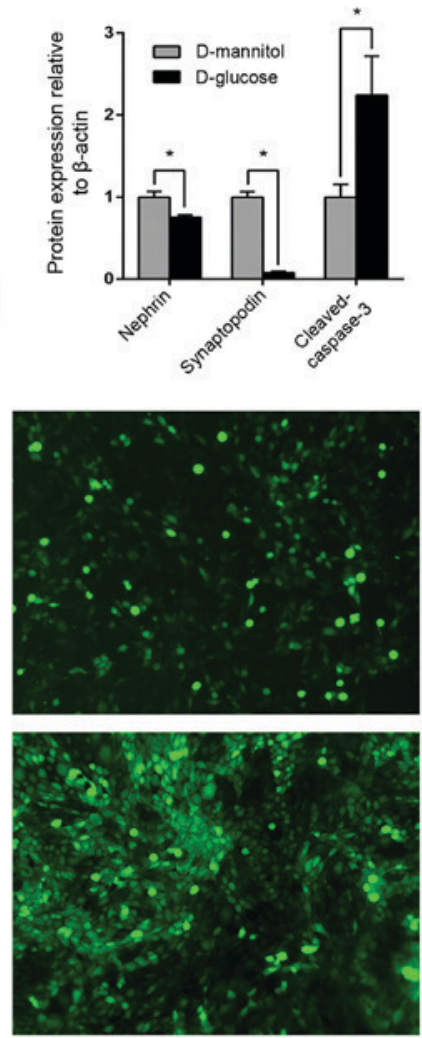

Figure 3. Mouse podocyte and renal mesangial cell impairment by high glucose concentrations. (A) Effect of high glucose on PGC-1 $\alpha$, PPRC1, PPAR $\gamma$, NRF1, NDUFS1 and COX4I1 mRNA expression. Mouse podocytes were cultured in $30 \mathrm{mM}$ glucose medium for 24 or $48 \mathrm{~h}$. Medium containing $5.5 \mathrm{mM}$ glucose with $24.5 \mathrm{mM}$ mannitol was applied as control. ${ }^{*} \mathrm{P}<0.05$ and ${ }^{\#} \mathrm{P}<0.01$, as indicated. (B) Western blot analysis of nephrin, synaptopodin and cleaved-caspase-3 protein levels in podocytes following treatment with $30 \mathrm{mM}$ glucose. (C) Quantification of average band intensity from four separate western blots. Data are presented as the mean \pm standard deviation $(n=3)$. "P<0.05, as indicated. (D) Levels of reactive oxygen species were detected using dichlorofluorescin diacetate and were observed with phase contrast bright-field (left panels) and epi-fluorescence (right panels) microscopy in SV40 MES 13 cells stimulated with either $30 \mathrm{mM}$ D-mannitol (top) or $30 \mathrm{mM}$ D-glucose (bottom) for $48 \mathrm{~h}$. Magnification, x400. PPAR $\gamma$, peroxisome proliferator-activated receptor $\gamma$; PGC-1 $\alpha$, PPAR $\gamma$ coactivator-1 $\alpha$; PPRC1, PGC-1-related coactivator; NRF1, nuclear respiratory factor 1; NDUFS1, NADH dehydrogenase (ubiquinone) Fe-S protein 1; COX4I1, cytochrome $\mathrm{C}$ oxidase subunit $4 \mathrm{I} 1$.

oxidase subunit 4I1 (COX4I1) of mitochondrial redox carriers in mouse podocytes were all significantly downregulated compared with the isotonic D-mannitol control (Fig. 3A). At the protein level, $48 \mathrm{~h}$ stimulation with $30 \mathrm{mM}$ glucose decreased the expression of specific podocyte marker proteins and increased cleaved-caspase 3 expression $(\mathrm{P}<0.05$; Fig. $3 \mathrm{~B}$ and $\mathrm{C}$ ) in mouse podocytes, indicating potential podocyte injury and apoptosis. Additionally, ROS levels were examined by DCF-DA fluorescence in cultured SV40 MES 13 mouse mesangial cells, and levels were markedly increased following stimulation with $30 \mathrm{mM}$ glucose for $48 \mathrm{~h}$ compared with the synchronous D-mannitol stimulation at the same concentration (Fig. 3D). These results indicate that high glucose may induce mitochondrial dysfunction in cultured podocytes and renal mesangial cells.

Effect of genetic regulation of PGC-1 $\alpha$ in high glucose-stimulated renal mesangial cells. The role PGC-1 $\alpha$ in renal mesangial cells in DKD was investigated by establishing cells with overexpression or knockdown of PGC-1 $\alpha$. PGC-1 $\alpha$ overexpression, 
A
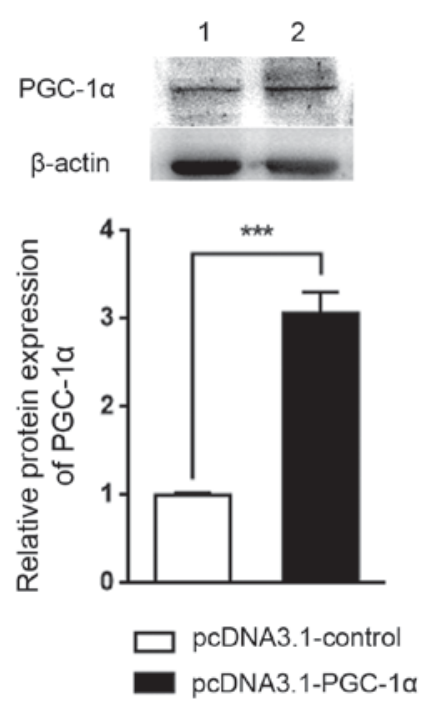

C

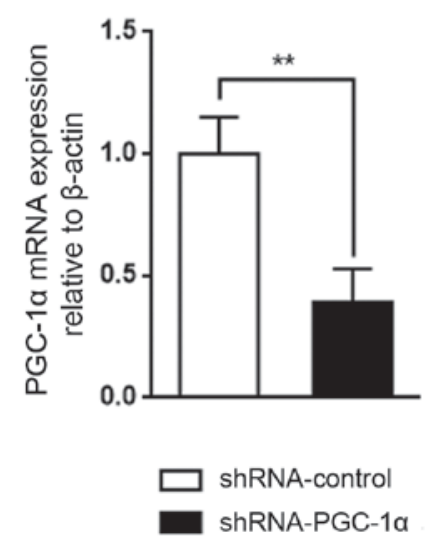

B

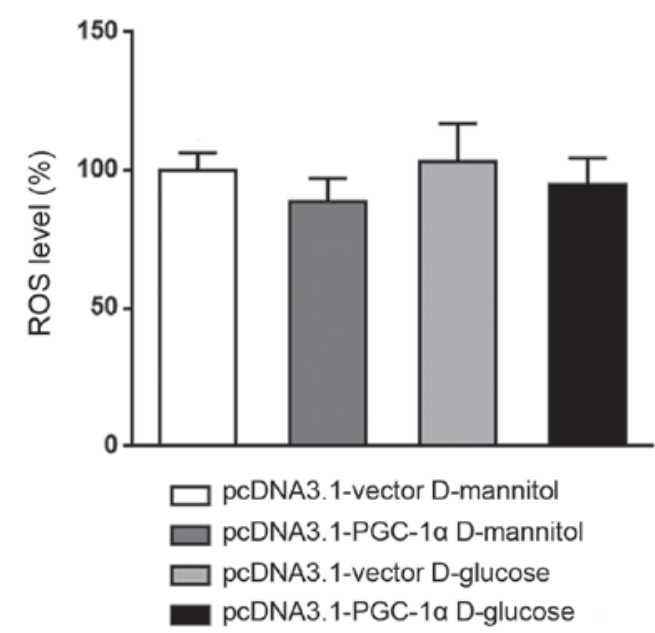

D

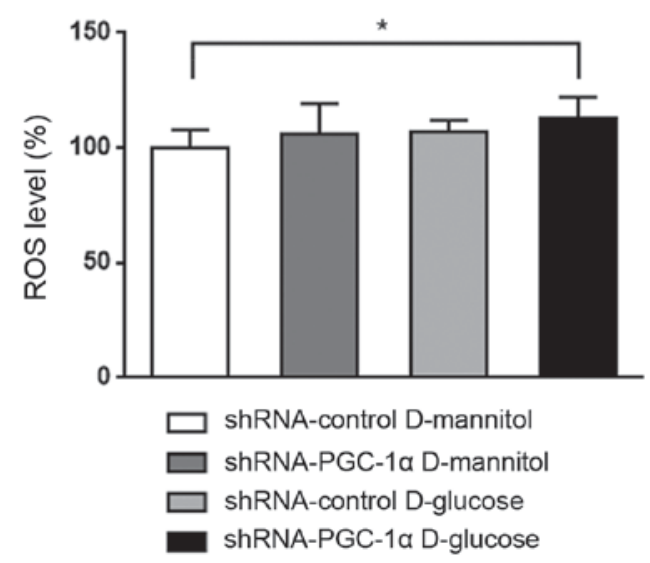

Figure 4. Effect of PGC-1 $\alpha$ knockdown or overexpression on high glucose-induced ROS generation in mesangial cells. (A) Confirmation of PGC-1 $\alpha$ expression in SV40 MES 13 cells transfected with pcDNA3.1-vector (lane 1) or pcDNA3.1-PGC-1 $\alpha$ (lane 2) by western blotting and the corresponding relative quantification of average band intensity. Data are presented as the mean \pm standard deviation $(n=3)$. (B) Intracellular ROS levels were detected in SV40 MES 13 cells following high glucose treatment in the PGC-1 $\alpha$ overexpression or control vector transfection groups using a fluorescence microplate reader. Data are presented as a percentage of the control group (pcDNA3.1-vector D-mannitol). The experiment was repeated three times ( $\mathrm{n}=6$ per group). (C) Confirmation of PGC-1 $\alpha$ mRNA expression by reverse transcription-quantitative polymerase chain reaction in SV40 MES 13 cells transduced with shRNA-control or shRNA-PGC-1 $\alpha(n=3)$. (D) ROS levels in SV40 MES 13 cells transduced with control or PGC-1 $\alpha$ shRNA and treated with D-glucose or D-mannitol were measured and presented as a percentage of the control group (shRNA-control D-mannitol) Three independent protein preparations were each run in 6 samples. All data are presented as the mean \pm standard deviation. ${ }^{*} \mathrm{P}<0.05,{ }^{* *} \mathrm{P}<0.01$ and ${ }^{* * *} \mathrm{P}<0.001$, as indicated. PGC-1 $\alpha$, peroxisome proliferator-activated receptor $\gamma$ coactivator-1 $\alpha$; ROS, reactive oxygen species; shRNA, short hairpin RNA.

which was confirmed by western blotting (Fig. 4A), appeared to reduce ROS levels in cultured SV40 MES 13 cells, although not significantly (Fig. 4B). By contrast, PGC-1 $\alpha$ knockdown, which was confirmed by RT-qPCR (Fig. 4C), with glucose treatment significantly increased ROS levels, compared with the shRNA-control D-mannitol group $(\mathrm{P}<0.05$; Fig. 4D). These results indicate that the downregulation of PGC-1 $\alpha$ may be a key step in DKD pathogenesis; PGC-1 $\alpha$ activation may lead to an antioxidative effect and may aid in halting DKD progression

\section{Discussion}

Rosiglitazone is a pharmacological agonist of PPAR $\gamma$ that promotes the expression of its coactivator, PGC-1 $\alpha$. As the bridge for communication between homeostasis and mitochondrial function, PGC-1 $\alpha$ induces NRF1 and NRF2 activation, which are nuclear-encoded transcription factors that promote the expression of multiple nuclear-encoded genes involved in the regulation of mitochondrial DNA transcription and the mitochondrial respiratory chain, leading to an overall reduction in ROS synthesis $(17,18)$.

Mitochondrial dysfunction and oxidative stress are considered to be major pathogenic mechanisms in DKD. Although PGC- $1 \alpha$ is a major nuclear factor that maintains mitochondrial dynamics and function, to the best of our knowledge, the effects and underlying mechanisms of PGC-1 $\alpha$ in DKD are yet to be elucidated. Recent findings have also indicated a protective role for PPARs in acute and chronic kidney disease, primarily through anti-inflammatory action $(19,20)$. However, only a limited number of studies have investigated the renoprotective effects of PPAR $\gamma$ agonists in regulating 
local and systemic metabolism through mitochondrial biogenesis $(19,20)$.

In the present study, increased levels of PGC- $1 \alpha$ in nuclear and cytosolic fractions were observed in the $\mathrm{db} / \mathrm{db}$ mouse model, and expression of E-cadherin was markedly reduced and COL1 $\alpha 1$ levels increased, indicating that EMT and renal cortex tissue fibrosis occurred in the $\mathrm{db} / \mathrm{db}$ mice models aged 14 weeks, and demonstrated pathological features of the early-stage DKD, determined as previously described (21). Additionally, rosiglitazone treatment induced PGC-1 $\alpha$ expression in nucleus and significantly ameliorated EMT and fibrosis compared with the DKD group. These findings indicate that PGC-1 $\alpha$ expression may be increased in the early stage of DKD as a compensatory response, as observed in cultured podocytes following glucose stimulation for $24 \mathrm{~h}$. Rosiglitazone promoted the protective effects of PGC- $1 \alpha$ against kidney fibrosis in DKD. In vitro and in vivo findings were consistent, as in vitro experiments on cultured SV40 MES 13 renal mesangial cells demonstrated that PGC-1 $\alpha$ knockdown increased glucose-induced ROS levels. Thus, PGC-1 $\alpha$ may have a protective role in oxidative stress and mitochondrial function.

In the current study, PGC-1 $\alpha$ upregulation was observed in DKD mice in vivo and was consistent with the observation that PGC-1 $\alpha$ mRNA expression was increased in mouse podocytes following glucose stimulation for $24 \mathrm{~h}$. However, this upregulation in mouse podocytes was reversed following a further $24 \mathrm{~h}$ stimulation. It is feasible that in the early stages of DKD, high glucose concentrations cause overloading of mitochondria, leading to the induction of PGC- $1 \alpha$ expression to enhance mitochondrial biogenesis and to reduce ROS synthesis, as a compensatory response. However, eventual decompensation leads to a more severe impairment in DKD. Upregulated PGC-1 $\alpha$ expression was previously reported in ATP depletion, exogenous ROS expression (22) and septicopyemia (23). In the present study, SV40 MES 13 renal mesangial cells stimulated with high glucose for $48 \mathrm{~h}$ exhibited a higher fluorescence intensity, indicating higher ROS levels compared with mannitol controls. However, this result was not observed in the subsequent overexpression and knockdown studies in SV40 MES 13 cells. Specifically, there was no significant difference detected in ROS levels following high glucose treatment in mesangial cells transfected with pcDNA3.1 vector or pLKO.1 shRNA control. This result may be due to the influence of transfection and infection, which has the potential to alter the susceptibility of the cells to high glucose concentrations.

Mitochondria are the major source of ROS in the majority of cell types (24). The results of the present study revealed that the expression of key mitochondrial respiratory chain complex components significantly decreased when diabetic podocytes were stimulated with high glucose, indicating potential mitochondrial impairment. Based on this data, it may be hypothesized that intracellular ROS was generated from the mitochondria to initiate multiple profibrotic responses (25), leading to a final outcome of kidney fibrosis in the context of diabetes.

In conclusion, the results of the current study indicate that the PPAR $\gamma$ agonist rosiglitazone may induce PGC- $1 \alpha$ expression to maintain mitochondrial function and to reduce
ROS generation. It may ameliorate podocyte impairment, GBM thickening and kidney fibrosis to aid in the prevention of DKD occurrence and progression. These results for rosiglitazone treatment in the present study indicated the potential ability of PPAR $\gamma$ agonists to be employed and developed as future DKD therapies.

\section{Acknowledgements}

The present study was supported by the National Key Research and Development Program of China (grant no. 2016YFC1305402), the National Natural Science Foundation of China (grant nos. 81270782 and 30771000), the Research Project of Science and Technology Commission of Shanghai Municipality (grant no. 15140902800), the Key Projects of National Basic Research Program of China 973 (grant no. 2012CB517701) and the National Key Technology R\&D Program (grant no. 2011BAI10B00).

\section{References}

1. Collins AJ, Foley RN, Chavers B, Gilbertson D, Herzog C, Johansen K, Kasiske B, Kutner N, Liu J, St Peter W, et al: United States Renal Data System 2011 Annual Data Report: Atlas of chronic kidney disease \& end-stage renal disease in the United States. Am J Kidney Dis 59 (1 Suppl 1): A7: e1-e420, 2012.

2. Zhang L, Long J, Jiang W, Shi Y, He X, Zhou Z, Li Y, Yeung RO, Wang J, Matsushita K, et al: Trends in chronic kidney disease in China. N Engl J Med 375: 905-906, 2016.

3. Kanwar YS, Wada J, Sun L, Xie P, Wallner EI, Chen S, Chugh S and Danesh FR: Diabetic nephropathy: Mechanisms of renal disease progression. Exp Biol Med (Maywood) 233: 4-11, 2008.

4. Abe Y, Sakairi T, Beeson C and Kopp JB: TGF- $\beta 1$ stimulates mitochondrial oxidative phosphorylation and generation of reactive oxygen species in cultured mouse podocytes, mediated in part by the mTOR pathway. Am J Renal Physiol 305: F1477-F1490, 2013.

5. Casalena G, Daehn I and Bottinger E: Transforming growth factor-beta, bioenergetics, and mitochondria in renal disease. Semin Nephrol 32: 295-303, 2012.

6. Liu C and Lin JD: PGC-1 coactivators in the control of energy metabolism. Acta Bioch Bioph Sin (Shanghai) 43: 248-257, 2011.

7. Handschin $C$ and Spiegelman BM: Peroxisome proliferator-activated receptor gamma coactivator 1 coactivators, energy homeostasis, and metabolism. Endocr Rev 27: 728-735, 2007.

8. Andersson U and Scarpulla RC: Pgc-1-related coactivator, a novel, serum-inducible coactivator of nuclear respiratory factor 1-dependent transcription in mammalian cells. Mol Cell Biol 21: 3738-3749, 2001.

9. Hickey FB, Corcoran JB, Docherty NG, Griffin B, Bhreathnach U, Furlong F, Martin F, Godson C and Murphy M: IHG-1 promotes mitochondrial biogenesis by stabilizing PGC-1 $\alpha$. J Am Soc Nephrol 22: 1475-1485, 2011.

10. Tran M and Parikh SM: Mitochondrial biogenesis in the acutely injured kidney. Nephron Clin Pract 127: 42-45, 2014.

11. Marmolino D, Manto M, Acquaviva F, Vergara P, Ravella A, Monticelli A and Pandolfo M: PGC-1alpha down-regulation affects the antioxidant response in Friedreich's ataxia. PLoS One 5: e10025, 2010

12. Miglio G, Rosa AC, Rattazzi L, Collino M, Lombardi G and Fantozzi R: PPARgamma stimulation promotes mitochondrial biogenesis and prevents glucose deprivation-induced neuronal cell loss. Neurochem Int 55: 496-504, 2009.

13. Wenz T, Diaz F, Spiegelman BM and Moraes CT: Activation of the PPAR/PGC-1alpha pathway prevents a bioenergetic deficit and effectively improves a mitochondrial myopathy phenotype. Cell Metab 8: 249-256, 2008.

14. Jin Y, Ratnam K, Chuang PY, Fan Y, Zhong Y, Dai Y, Mazloom AR, Chen EY, D'Agati V, Xiong H, et al: A systems approach identifies HIPK 2 as a key regulator of kidney fibrosis. Nat Med 18: 580-588, 2012. 
15. Zhang Z, Zhang H, Li B, Meng X, Wang J, Zhang Y, Yao S, Ma Q, Jin L, Yang J, et al: Berberine activates thermogenesis in white and brown adipose tissue. Nat Commun 5: 5493, 2014.

16. Livak KJ and Schmittgen TD: Analysis of relative gene expression data using real-time quantitative PCR and the 2(-Delta Delta C(T)) method. Methods 25: 402-408, 2001

17. Weinberg JM: Mitochondrial biogenesis in kidney disease. J Am Soc Nephrol 22: 431-436, 2011.

18. Ishimoto $\mathrm{Y}$ and Inagi R: Mitochondria: A therapeutic target in acute kidney injury. Nephrol Dial Transpl 31: 1062-1069, 2016.

19. Ruan X, Zheng F and Guan Y: PPARs and the kidney in metabolic syndrome. Am J Physiol Renal Physiol 294: F1032-F1047, 2008.

20. Wang XX, Jiang T and Levi M: Nuclear hormone receptors in diabetic nephropathy. Nat Rev Nephrol 6: 342-351, 2010.

21. Sharma K, McCue P and Dunn SR: Diabetic kidney disease in the db/db mouse. Am J Physiol Renal Physiol 284: F1138-F1144, 2003.
22. St-Pierre J, Drori S, Uldry M, Silvaggi JM, Rhee J, Jäger S, Handschin C, Zheng K, Lin J, Yang W, et al: Suppression of reactive oxygen species and neurodegeneration by the PGC-1 transcriptional coactivators. Cell 127: 397-408, 2006.

23. Haden DW, Suliman HB, Carraway MS, Welty-Wolf KE, Ali AS, Shitara H, Yonekawa $\mathrm{H}$ and Piantadosi CA: Mitochondrial biogenesis restores oxidative metabolism during Staphylococcus aureus sepsis. Am J Resp Crit Care 176: 768-777, 2007.

24. Lambert AJ and Brand MD: Reactive oxygen species production by mitochondria. Methods Mol Biol 554: 165-181, 2009.

25. Coughlan MT and Sharma K: Challenging the dogma of mitochondrial reactive oxygen species overproduction in diabetic kidney disease. Kidney Int 90: 272-279, 2016.

This work is licensed under a Creative Commons Attribution-NonCommercial-NoDerivatives 4.0 International (CC BY-NC-ND 4.0) License. 